\title{
Reply to: Relationship between LAT1 expression and resistance to chemotherapy in pancreatic ductal adenocarcinoma
}

\author{
Kyoichi Kaira $^{1}$
}

Received: 15 May 2018 / Accepted: 15 June 2018 / Published online: 25 June 2018

๑) Springer-Verlag GmbH Germany, part of Springer Nature 2018

Weng et al. pointed out the problem of statistical analysis. However, Cox's proportional hazards assumption was performed according to the procedure of JMP Pro 12.0 software (SAS Institute Inc., Cary, NC, USA). Thus, there are no serious problems as described by authors.

To do multivariate survival analysis, some appropriate factors are chosen by several methods. The established methods to choose the clinicopathological factors in the univariate analysis remain unclear. According to previous reports, it is not a problem that the clinicopathological factors with $p$ value less than 0.05 in the analysis were chosen for the multivariate analysis.

\section{Compliance with ethical standards}

Conflict of interest KK declares that he has no conflict of interest.

Ethical approval This article does not contain any studies with human participants performed by any of the authors.
Kyoichi Kaira

kkaira1970@yahoo.co.jp

1 Department of Oncology Clinical Development, Gunma University Graduate School of Medicine, Showa-machi, Maebashi, Gunma 371-8511, Japan 